Cahiers $d u$ MONDE RUSSE

\section{Cahiers du monde russe}

Russie - Empire russe - Union soviétique et États indépendants

$52 / 1 \mid 2011$

Varia

\title{
Un anti-Gide allemand : Lion Feuchtwanger
}

Lion Feuchtwanger, a German opponent to Gide

\section{Anne Hartmann}

\section{(2) OpenEdition \\ Journals}

Édition électronique

URL : https://journals.openedition.org/monderusse/9323

DOI : $10.4000 /$ monderusse. 9323

ISSN : $1777-5388$

Éditeur

Éditions de l'EHESS

Édition imprimée

Date de publication : 5 mars 2011

Pagination : 109-132

ISBN : 978-2-7132-2351-8

ISSN : $1252-6576$

Référence électronique

Anne Hartmann, "Un anti-Gide allemand : Lion Feuchtwanger », Cahiers du monde russe [En ligne], 52/1 | 2011, mis en ligne le 28 mars 2014, consulté le 02 septembre 2022. URL : http://

journals.openedition.org/monderusse/9323; DOI : https://doi.org/10.4000/monderusse.9323 


\title{
ANNE HARTMANN \\ UN ANTI-GIDE ALLEMAND
}

\author{
Lion Feuchtwanger*
}

Ce qui distingue peut-être le plus Lion Feuchtwanger de ses compagnons d'infortune, c'est qu'il a su s'adapter à l'émigration : il n'était pas amer, il ne se plaignait ni de l'incertitude de son existence, ni des privations, ni de la «nostalgie du cœur en exil, du déracinement, de l'angoisse nerveuse de se trouver apatride $»^{1}$. Et pourtant, il aurait eu largement matière à se plaindre : auteur juif allemand de renommée mondiale grâce à ses romans Jud Süß (1925; version française : Le Juif Süss, 1929), Erfolg (1930) et Der jüdische Krieg (1932 ; version française : La Guerre des Juifs, 1933), il se trouvait justement aux États-Unis pour donner une série de conférences quand il eut connaissance de l'arrivée au pouvoir d'Hitler le 30 janvier 1933. Peu de temps après, des troupes SA saccageaient sa villa de Berlin et le 10 mai, ses livres étaient brûlés sur le premier autodafé nazi. Sur la première liste d'expatriation publiée par le journal Reichsanzeiger en août, le nom de Feuchtwanger figurait à la sixième place - une distinction honorable qui signifiait cependant le retrait de son titre de docteur et de sa nationalité, la confiscation de ses biens et, surtout, la perte de sa bibliothèque et de manuscrits précieux. Feuchtwanger ne retourna jamais plus en Allemagne ${ }^{2}$.

Il s'établit à Sanary-sur-Mer, un village de pêcheurs situé dans le sud de la France et qui devint peu à peu, avec Nice et Paris, la « capitale de la littérature allemande » en exil (Ludwig Marcuse). Alors âgé de 49 ans, Feuchtwanger y commença une

* J'adresse mes vifs remerciements à Monsieur Michel Aucouturier qui a, dès le début, soutenu et accompagné la rédaction de cet article.

1. Thomas Mann, «Warum ich nicht zurückkehre », in Johannes Franz Gottlieb Grosser, éd., Die große Kontroverse: Ein Briefwechsel um Deutschland, Hamburg [et al.] : Nagel, 1963 , p. 28.

2. À propos de la biographie voir en particulier : Volker Skierka, Lion Feuchtwanger: Eine Biographie, Berlin : Quadriga, 1984 ; Wilhelm von Sternburg, Lion Feuchtwanger: Ein deutsches Schriftstellerleben, Berlin - Weimar : Aufbau, 1994. 
nouvelle vie qui devait durer quasiment sept ans. Sa maison, aménagée avec soin par sa femme Marta, devint un lieu de rencontre politique et littéraire : des voisins (intermittents) du sud de la France, comme Thomas et Heinrich Mann, Ludwig Marcuse, Erwin Piscator, Ernst Toller, Friedrich Wolf, Ernst Bloch et d'autres émigrés allemands sont passés chez les Feuchtwanger ; Arnold Zweig et Bertolt Brecht y venaient presque tous les ans. Ce n'était assurément pas une parfaite idylle : les relations entre les intellectuels étaient souvent affectées par des rivalités littéraires, des divergences politiques et aussi la concurrence économique dues à l'exil. Feuchtwanger put tout de même jouir d'une vie confortable là-bas, sur la côte méditerranéenne, avant de subir une nouvelle expulsion : en 1939-1940, Feuchtwanger fut interné deux fois au camp des Milles en tant que « ressortissant de pays ennemi »; il parvint de justesse à s'enfuir aux États-Unis ${ }^{3}$.

Contrairement à la plupart de ses collègues, Feuchtwanger eut aussi du succès à l'étranger. Il sut rester un auteur productif (il écrivit au total cinq romans durant son exil en France) et ses œuvres lui permettaient de mener une vie aisée, notamment grâce à sa popularité dans les pays anglo-saxons. Travailler assidûment à ses œuvres littéraires, passer ses soirées dans les casinos sur la Riviera, séjourner à Paris ou à Londres pour négocier avec ses éditeurs, participer à des congrès divers ou se payer le luxe d'un bon hôtel avec une amie : voilà ce qu'ont été pour Feuchtwanger les années passées en France. Même si son sens des affaires et son train de vie suscitaient pas mal de jalousie et qu'il était qualifié « d'écrivain de divertissement » ou «d'industriel de la littérature », Feuchtwanger était en général apprécié par ses collègues pour son flegme, son optimisme et sa générosité. «Vous êtes le seul homme que je connaisse dont j'oserais dire qu'il est heureux », résumait plus tard son voisin de Sanary, l'écrivain et philosophe Ludwig Marcuse ${ }^{4}$. D'après lui, Feuchtwanger savait se protéger par quatre remparts : son assurance inébranlable, nourrie par son succès et qui l'immunisait contre tout doute et tout ressentiment ; la fierté de son appartenance au peuple juif, le peuple civilisé le plus ancien ; « une weltanschauung inébranlable et peu ambiguë » qui reposait sur la foi en la raison ; et la forteresse de livres qu'il avait rassemblée à Sanary et qui lui permettait de vivre au sens propre « dans la littérature du monde $»^{5}$.

Il n'y avait qu'une seule scène sur laquelle Feuchtwanger devait finalement échouer, la scène politique internationale, et ce de manière même tragique. De par son engagement contre le fascisme, il se tourna de plus en plus vers l'autre mouvement totalitaire, le stalinisme. Son récit de voyage, Moskau 1937, s'achève avec trois « oui » en faveur de l'Union soviétique, incluant Stalin et les

3. Son récit sur l'internement et la fuite fut publié pour la première fois en 1942 sous le titre de Unholdes Frankreich, les éditions suivantes furent intitulées Der Teufel in Frankreich (version française : Le diable en France, 1985).

4. Lettre de Marcuse à Feuchtwanger (été 1944), in Harold von Hofe, Sigrid Washburn, éds., Lion Feuchtwanger: Briefwechsel mit Freunden 1933-1958, Vol. II, Berlin et Weimar : Aufbau, 1991, p. 18.

5. Ludwig Marcuse, Mein zwanzigstes Jahrhundert: Auf dem Weg zu einer Autobiographie, Zurich : Diogenes, 1975,p. 185. 
procès-spectacle : «Après toute l'hypocrisie de l'ouest, cela fait du bien de voir une œuvre à laquelle on ne peut que dire Oui, Oui, Oui de tout son cœur. Et c'est parce qu'il me semblait indécent de garder ce oui dans la gorge que j'écrivis ce livre. ${ }^{6}$ Cette confession provoqua un scandale dans le monde de la littérature et des intellectuels. Le petit ouvrage porta un préjudice si durable à la réputation de Feuchtwanger que, jusqu'à aujourd'hui, il a été soit marginalisé par les chercheurs, soit considéré comme le point central de sa personnalité littéraire. Les critiques qualifient l'attitude de Feuchtwanger d'aveuglement, voire pire : le fait qu'il fasse l'apologie du stalinisme révélerait la perte de tout jugement moral et politique ${ }^{7}$.

Du point de vue de sa biographie, il était tout à fait logique que Feuchtwanger, le juif exilé, combatte Hitler avec passion depuis le début. Aussi bien dans ses romans historiques que dans ceux traitant de l'époque contemporaine, la préoccupation principale de Feuchtwanger était de dénoncer le « retour de la barbarie en Allemagne » et sa « victoire temporaire sur la raison $»^{8}$, et de tenter de les contrecarrer. Dans Erfolg, il avait déjà dépeint l'avancée du mouvement national sur un terrain favorable à l'imbécillité et à la bêtise dans la province bavaroise. Die Geschwister Oppermann (1933 ; version française: Les Oppermann, 1934) illustre, à travers le destin de trois frères issus d'une famille juive connue, le triomphe du régime national-socialiste ainsi que l'échec de la bourgeoisie allemande. Ce roman, jugé par Klaus Mann comme étant « la représentation narrative de la tragédie allemande la plus lue et ayant le plus grand impact $»^{9}$, atteignit un nombre d'éditions considérable, fut traduit dans plusieurs langues et adapté pour le cinéma en URSS. Dans son roman satirique Der falsche Nero (1936 ; versions françaises : Néron l'imposteur, 1984 / Le faux Néron, 1995), Feuchtwanger décrit, transposée dans l'Antiquité, la montée d'Hitler et de ses vassaux et anticipe en même temps leur chute.

Feuchtwanger ne se sentait nullement lié à un parti ou une idéologie, seulement au progrès historique et aux Lumières. Ses héros, fréquemment des marginaux, sont souvent pris entre ascension et échec, action et réflexion, raison et impulsivité, nationalisme et internationalisme, génie artistique et société. Feuchtwanger

6. Lion Feuchtwanger, Moskau 1937: Ein Reisebericht für meine Freunde, Amsterdam : Querido, 1937,p. 153.

7. Cf. en particulier Karl Kröhnke, Lion Feuchtwanger - Der Ästhet in der Sowjetunion: Ein Buch nicht nur für seine Freunde, Stuttgart : Metzler, 1991. Pour la critique actuelle voir notamment Daniel Azuélos, « Lion Feuchtwanger ou l'intellectuel bourgeois prisonnier de ses contradictions », in Idem, éd., Lion Feuchtwanger et les exilés de langue allemande en France de 1933 à 1941, Bern : Peter Lang, 2006, p. 49-59 ; Feliks Gimel’farb, « “Agitpunkt” Stalina. K 70-letiju vyhoda knigi L. Fejhtvangera Moskva 1937 [“Agitpunkt” de Stalin. À l'occasion du $70^{\mathrm{e}}$ anniversaire de la publication du livre de L. Feuchtwanger Moscou 1937] », Evrejskaja gazeta, février 2007 ; Martin Mauthner, « "I Came, I Saw, I Shall Write”. Feuchtwanger's Misguided Mission to Moscow » in Idem, German Writers in French Exile 1933-1940, London [et al.] : Vallentine Mitchell, 2007, p. 165-181; Michail Ryklin, Kommunismus als Weltreligion : Die Intellektuellen und die Oktoberrevolution, Frankfurt/M. - Leipzig : Verlag der Weltreligionen, 2008, p. 149-159; Wolfgang Geier, Wahrnehmungen des Terrors: Berichte aus Sowjetrussland und der Sowjetunion, 1913-1938, Wiesbaden : Harrassowitz, 2009, p. 85-94.

8. Lion Feuchtwanger, « Nachwort », in Idem, Exil: Roman, Amsterdam : Querido, 1940, p. 983.

9. Klaus Mann, Der Wendepunkt: Ein Lebensbericht, Reinbek : Rowohlt, 1984, p. 319. 
considérait que l'écrivain devait être au-dessus des classes et garder une certaine distance par rapport aux événements : «Celui qui agit est toujours sans scrupules. Seul a conscience celui qui observe », affirmait Feuchtwanger, répétant ainsi une maxime de Goethe ${ }^{10}$.

Toutefois, au milieu des années 30, il lui arriva de temps à autre de s'écarter de ce principe. Il faisait partie des fondateurs du « Schutzverband Deutscher Schriftsteller im Exil [Association de défense des écrivains allemands en exil]»; il fut l'initiateur, avec entre autres André Gide, Romain Rolland et H.G. Wells, de la « Deutsche Freiheitsbibliothek [Bibliothèque allemande de la liberté] »; en 1935, il tint un discours à Paris lors du célèbre « Congrès international des écrivains pour la défense de la culture » et il signa des pétitions en faveur d'un front populaire allemand, activités qui dénotent un rapprochement croissant avec l'Union soviétique. Plus la déception vis-à-vis d'Hitler augmentait face à la politique que tenaient les démocraties occidentales, plus l'espoir de voir l'URSS s'engager dans le combat contre le national-socialisme grandissait, en particulier lorsque l'Union soviétique vint en aide à la république espagnole. Au cours des années 1930 apparut une culture antifasciste particulière qui remplaça et paracheva les étapes précédentes de la fascination pour la Russie que nourrissaient la promesse quasiment mythique de la révolution d'Octobre et l'utopie de l'ordre et du progrès incarnée par l'économie planifiée ${ }^{11}$. Une nouvelle source d'attraction s'y ajouta pour les écrivains : en URSS, la littérature jouissait d'un prestige dont elle était largement dépourvue en Occident.

L'invitation d'intellectuels renommés dont la parole avait un certain poids en Occident était un élément important de la politique soviétique en matière de culture, révisée vers le milieu des années 1930, au moment où l'on rejetait la lutte de classes et l'ouvriérisme au profit d'une perspective de large rassemblement et d'une esthétique de la représentation. On attachait de l'importance aux visites de voyageurs éminents - et ces visites étaient tout aussi soigneusement préparées, accompagnées et évaluées par les Soviétiques ${ }^{12}$. Feuchtwanger avait déjà été courtisé avec insistance avant et

10. Même à Moscou il défendit cette maxime. Cf. « Redemanuskript für einen Schriftstellerempfang » [sans date]. Feuchtwanger Memorial Library (FML). Special Collections. University of Southern California (USC), Los Angeles : Box D 10-a (Articles and information about LF's trip to USSR) [dans ce qui suit: Feuchtwanger Articles].

11. Sur les étapes différentes voir François Furet, Le passé d'une illusion, P. : Robert Laffont, 1995.

12. À propos de la diplomatie culturelle soviétique, leur institutions et instruments, il y a de nombreuses recherches ; parmi les publications actuelles, cf. Rachel Mazuy, Croire plutôt que voir ? Voyages en Russie soviétique (1919-1939), P : Odile Jacob, 2002 ; Jean-François Fayet, «Entre culture, politique et lobyying diplomatique : la Société pansoviétique d'échanges culturels avec l'étranger », in Hans Ulrich Jost, Stéfanie Prezioso, éds., Relations internationales, échanges culturels et réseaux intellectuels, Lausanne : Antipodes, 2002, p. 97-113 ; J.-F. Fayet, «La société pour les échanges culturels entre 1'URSS et l'étranger (VOKS) », Relations Internationales, 115, 2003, p. 411-423 ; Matthias Heeke : Reisen zu den Sowjets. Der ausländische Tourismus in Russland 1921-1941, Münster : Lit-Verlag, 2003 ; A.V. Golubev, « ... vzgljad na zemlju obetovannuju »: Iz istorii sovetskoj kul'turnoj diplomatii 1920-1930-h godov [« ... un regard sur la terre promise ». De l'histoire de la diplomatie culturelle soviétique dans les années 1920-1930], M. : Institut rossijskoj istorii RAN, 2004; Leonid Maksimenkov, « Očerki nomenklaturnoj istorii sovetskoj literatury. Zapadnye piligrimy u stalinskogo prestola (Fejhtvanger i drugie) [Esquisses de l'histoire de la nomenklatura littéraire soviétique. Pèlerins occidentaux 
durant le congrès parisien : « Les Russes sont particulièrement agréables avec moi », écrit-il le 24 juin 1935 dans son journal intime, et le lendemain : «Ce sont surtout les Russes qui encensent mes œuvres. Gorki aurait recommandé Erfolg avec de fortes louanges $»^{13}$. Suivirent des lettres élogieuses et des compliments ( $«$ En Russie, je suis sans conteste reconnu comme étant le plus grand écrivain étranger $\left.»^{14}\right)$, des panégyriques de l'écrivain dans des revues soviétiques, et même une « soirée Feuchtwanger » en l'honneur de l'écrivain dans la Maison des savants de Moscou (le $1^{\text {er }}$ juin 1936). Le projet, prévu depuis longtemps, d'aller en Union soviétique («Vous pouvez aussi travailler ici. On peut même mettre tout un palais à votre disposition en Crimée ou dans le Caucase $\gg^{15}$ ) commençait peu à peu à voir le jour. Pour la première fois, et à sa grande surprise, l'écrivain recevait des rémunérations généreuses pour ses œuvres éditées en URSS. Feuchtwanger montra qu'il méritait cette gratification en écrivant des articles dans la presse soviétique, entre autres une polémique contre Knut Hamsun et un éloge de la nouvelle constitution soviétique ${ }^{16}$.

En 1936, Feuchtwanger prit en charge avec Bertolt Brecht (au Danemark) et Willi Bredel (à Moscou) la publication de la revue allemande Das Wort (Le mot) publiée à Moscou avec l'approbation du Politburo. C'est Feuchtwanger qui avait proposé ce titre. Mihail Kol'cov, directeur des éditions «Žurgaz », correspondant spécial de la Pravda, émissaire en Espagne et responsable de la Commission

aux pieds de Stalin (Feuchtwanger et autres)] », Voprosy literatury, 2, 2003, p. 241-297 et 3, 2004, p. 274-353; Ludmila Stern, Western Intellectuals and the Soviet Union, 1920-40: From Red Square to the Left Bank, London - New York : Routledge, 2007; G.B. Kulikova, «Sovetskie voždi i zarubežnye vizitery [Les dirigeants soviétiques et les visiteurs étrangers] », in A.V. Golubev, éd., Rossija i mir glazami drug druga: Iz istorii vzaimovosprijatija [La Russie et le monde par le regard de l'un et de l'autre. De l'histoire de la perception mutuelle], Vyp. 4, M. : Institut Rossijskoj Istorii RAN, 2007, p. 80-105; Sheila Fitzpatrick, « Foreigners observed: Moscow Visitors in the 1930s under the Gaze of their Soviet Guides », Russian History 35 (1-2), 2008, p. 215-234; Anne Hartmann, « Literarische Staatsbesuche. Prominente Autoren des Westens zu Gast in Stalins Sowjetunion », in Siegfried Ulbrecht, Helena Ulbrechtová, éds., Die Ost-West-Problematik in den europäischen Kulturen und Literaturen. Ausgewählte Aspekte. Problematika Východ - Západ v evroských kulturách a literaturách. Vybrané aspekty. Praha : Slovanský ústav AV ČR et Dresden : Neisse, 2009, p. 229-275; Eva Oberloskamp, Fremde neue Welten: Reisen deutscher und französischer Linksintellektueller in die Sowjetunion 1917-1939, München : Oldenbourg Verlag, 2011 ; Michael David-Fox, Showcasing the Great Experiment: Cultural Diplomacy and Western Visitors to Soviet Russia, 1921-1941, Oxford - New York : Oxford University Press, 2011.

13. FML, Special Collections, Box 19-b: Diaries transcriptions 1906-1940.

14. Ibid., 20 mai1936.

15. Lettre de Maria Osten à Lion Feuchtwanger, 19.4.1936. RGALI (Rossijskij Gosudarstvennyj Arhiv Literatury i Iskusstva - Archives d'État de Russie de littérature et d'art), Moscou, f. 631, op. 13, d. 65. Elsa Triolet, la femme d'Aragon, se plaint dans une lettre du 10 novembre [1936] à Vsevolod Višnevskij du fait que Gide ait pu écrire sans surveillance (« besprizornyj Gide ») « un livre extrêmement répugnant sur l'Union ». (in T.V. Balašova, éd., Dialog pisatelej : Iz istorii russko-francuzskih kul'turnyh svjazej XX veka 1920-1970 [Dialogue d'écrivains. De l'histoire des liens culturels franco-russes au Xx ${ }^{\mathrm{e}}$ siècle], M. : IMLI RAN, 2002, p. 761) - cela implique, qu'en présence et sous la surveillance des «mouchards », le résultat aurait été différent.

16. « Licemerie i besstydstvo. Pis'mo Liona Fejhtvangera [Hypocrisie et impudeur. Lettre de Lion Feuchtwanger] », Literaturnaja gazeta, 1.5.1936; «Pri čtenii proekta Sovetskoj konstitucii [En lisant le projet de la constitution soviétique] », Pravda, 1.7.1936. 
étrangère de l'Union des écrivains soviétiques, s'occupait de l'aspect rédactionnel et du financement de la revue. C'est surtout la compagne allemande de Kol'cov, Maria Osten, qui se chargeait à ses côtés des contacts entre l'est et l'ouest. Das Wort devait devenir l'organe représentatif du front populaire allemand, mais dès le début (le premier numéro parut en juillet 1936) la tâche se révéla difficile : la communication entre les éditeurs et les contributeurs était souvent ardue ; Bredel se sentait isolé à Moscou avec son travail ; le non-versement des honoraires et les retards dans la distribution lui posaient problème.

Mais ce qui aggrava vraiment les choses pour la revue ainsi que pour le front populaire en général, ce fut l'ébranlement déclenché par le premier procèsspectacle de Moscou avec la condamnation à mort des seize membres d'un soidisant «centre trotskiste-zinov'eviste » en août 1936. Par la suite de ce verdict, l'intellectuel suisse de gauche Rudolf Jakob Humm adressa une lettre ouverte à la rédaction de Das Wort à Moscou, déclarant « tirer un trait entre lui et l'Union soviétique $\gg^{17}$. L'écrivain italien Ignazio Silone demanda lui aussi que son nom cesse d'apparaître dans la revue. Selon lui, la solidarité avec la Russie impliquait le consentement à l'emploi de méthodes arbitraires contre les opposants (déportations, procédures judiciaires douteuses, meurtres) : «Quelle valeur ont donc toutes vos protestations contre la police et les tribunaux fascistes ? [...] Quelle est la valeur morale de cet humanisme que vous prétendez incarner ? ${ }^{18}$

Le 8 novembre, depuis Paris, Maria Osten rapporte à Willi Bredel, basé à Moscou : «Ici, toute la presse a divulgué le refus de Silone à Das Wort », et d'annoncer encore un autre scandale :

Ici le climat entre les intellectuels est assez tendu. Il y a trois jours, Gide a publié la préface de son livre sur la Russie. C'est tout simplement effarant : c'est tout simplement la confession d'un trotskiste. Tu peux t'imaginer combien nous sommes furieux et abattus. Aragon voulait lui en parler parce qu'on chuchotait déjà dans son dos, mais Gide l'a mis à la porte sans cérémonie..$^{19}$

Peu après, parut le récit de voyage d'André Gide Retour de l'U.R.S.S. Même si Gide ne signait nullement là sa rupture avec le communisme, auquel il s'était intéressé depuis le début des années 30, la presse de gauche proche du parti ne vit ni les compliments ni la curiosité pour la vie en URSS, mais seulement la critique qu'elle considéra comme une trahison de la cause antifasciste ${ }^{20}$.

17. Volksrecht (Zurich), 27.8.1936 ; cité par Peter Huber, Stalins Schatten in die Schweiz. Schweizer Kommunisten in Moskau. Verteidiger und Gefangene der Komintern, Zurich : Chronos, 1994, p. 70.

18. Arbeiter Zeitung (Bâle), 24.9.1936; cité par Denunziation und Verrat. Die 30er Jahre in der Sowjetunion (= europäische ideen 79). Berlin : Zimmermann, 1992, p. 21-23.

19. Lettre de Maria Osten à Willi Bredel, Paris, 8.11.1936. RGALI, f. 631, op. 12, d. 143.

20. À propos de l'hommage et de la critique de Gide dans les médias soviétiques, cf. Alain Goulet, « Gide à travers la presse soviétique de 1932 à 1937 », La Revue des lettres modernes, 223-227, 1970, p. 136-178; à propos des réactions en France cf. Rudolf Maurer, André Gide et l'URSS, Berne : Tillier, 1983, p. 129-148. 
Feuchtwanger prit le récit de voyage de Gide fraîchement publié pour le lire dans le train qui devait l'amener à Vienne, Varsovie, puis Moscou le 27 novembre 1936. Cependant, ce n'est pas seulement le livre, mais également l'ombre de Gide qui allait l'accompagner tout au long de son voyage. Pour les responsables de Moscou, la «Société pansoviétique pour les relations culturelles entre l'URSS et l'étranger » (Vsesojuznoe obščestvo kul'turnoj svjazi s zagranicej, VOKS), la Commission étrangère de l'Union des écrivains soviétiques, les services secrets, et bien d'autres encore, le résultat du voyage de Gide constituait une débâcle qui ne devait en aucun cas se reproduire.

La préoccupation que reflètent les rapports internes de ces organismes n'en est que plus grande : le 7 décembre, Boris Tal', chef du département de la presse et de l'édition du Comité central, informa Stalin qu'il faudrait être extrêmement attentif et se tenir sur ses gardes, qu'il serait nécessaire de mettre quelqu'un derrière Feuchtwanger, une personne à laquelle l'auteur allemand faisait confiance, et de recruter des gens éprouvés et prudents pour le «travail technique », c'est-à-dire la traduction $^{21}$. On ne peut exclure que la rencontre avec Stalin accordée à Feuchtwanger le 8 janvier 1937 ait fait partie des mesures visant à éviter les erreurs commises dans l'encadrement de Gide. Celui-ci, en effet, qui pourtant avait attendu sa rencontre avec Stalin avec « grande impatience », n'avait pu obtenir une entrevue ${ }^{22}$.

La tâche décrite par Tal', qui consistait à organiser et contrôler l'entourage de l'écrivain, fut certainement plus facile dans le cas de Feuchtwanger, qui resta durant ses dix semaines en Russie soit à Moscou, soit dans des maisons de repos des environs. Plus Gide s'éloignait de la capitale et se dirigeait vers le Caucase, moins il avait été encadré, mais le voyage n'en avait été que plus édifiant ${ }^{23}$. En outre, les membres de son groupe de voyageurs qui parlaient russe (Jeff Last, Jacques Schiffrin) et connaissaient la situation en Russie (Pierre Herbart avait travaillé pendant huit mois pour la revue Littérature internationale à Moscou à partir de novembre 1935) avaient pu compléter et relativiser les impressions de Gide ainsi que les propos de ses interlocuteurs soviétiques ${ }^{24}$.

Feuchtwanger non plus ne voyageait pas seul (il était entre autres accompagné par sa maîtresse Eva Herrmann) mais il dépendait complètement de ses interprètes pour la compréhension et la communication. En la personne de Dora Karavkina on lui assigna, comme Tal' l'avait requis, une interprète chevronnée ${ }^{25}$. Elle s'occupait

21. Cf. le dossier volumineux sur le séjour de Feuchtwanger à Moscou dans RGASPI (Rossijskij gosudarstvennyj arhiv social'no-političeskoj istorii - Archives d'État de Russie d'histoire socio-politique), Moscou, f. 558, op. 11, d. 820.

22. «Kol'cov Stalinu s pros’boj o prieme Andre Žida [Lettre de Kol'cov à Stalin le priant de recevoir André Gide] », 8.7.1936, in L.V. Maksimenkov, éd., Bol`šaja cenzura : Pisateli i žurnalisty $v$ strane sovetov 1917-1956 [La grande censure : Écrivains et journalistes aux pays des soviets, 1917-1956], M. : Materik, 2005, p. 422. Si un long entretien se révélait impossible, alors peutêtre une brève rencontre au théâtre ou dans un autre lieu. Un refus irriterait fortement Gide.

23. Cf. André Gide, Retouches à mon Retour de 1'U.R.S.S., P. : Gallimard, 1937, p. 75.

24. Cf. Ibid., p. 71-74. Pour les détails sur le voyage, cf. Maurer, André Gide, p. 85-117.

25. On sait peu de choses sur son origine et sa carrière, mais Dora L'vovna Karavkina (16 janvier 1896 à Kaunas - 7 août 1977 à Vilnius) faisait sans aucun doute partie des meilleures spécialistes de la langue allemande de la VOKS : elle avait également accompagné le célèbre 
du planning de Feuchtwanger et veillait à ce qu'il soit satisfait ; elle se considérait en même temps comme son mentor bolchevik, chargé de corriger ses opinions erronées. Ses rapports ${ }^{26}$ dépeignent un hôte difficile et exigeant : l'écrivain se plaignait du manque de confort, mais aussi du bas niveau de vie des Soviétiques, du conformisme social, du manque de liberté d'expression et de l'état peu gratifiant de la littérature. Par cette critique, Feuchtwanger se rapprochait de Gide et, d'après Karavkina, il l'aurait même défendu à plusieurs reprises. Il commenta les défauts de l'hôtel Métropole en disant que « ces quelques détails dans l'organisation avaient dû agir sur l'humeur d'André Gide, un homme susceptible et artiste (čeloveka nervnogo, hudožnika) ${ }^{27}$. Une autre fois, Feuchtwanger déclara, fâché par le remaniement d'un discours qui durait des heures, « qu'il était "dangereux" de donner son avis chez nous » et ajouta « qu'on voyait bien comment cela s'était passé avec Gide $»^{28}$. Comme Karavkina, d'autres observateurs aussi craignirent que la visite de Feuchtwanger n'aboutît à un second fiasco, comme le suggérait un poème satirique qui circulait à Moscou à ce moment-là : «Fejhtvanger u dverej / stoit s umil'nym vidom ; / o kak by sej Evrej / ne pokazalsja Židom. » L'émigré autrichien Hugo Huppert, dans son journal intime du 30 janvier 1937, releva dans un discours de Vsevolod Višnevskij devant l'Union des écrivains

une polémique enragée à l'égard de Feuchtwanger, « porteur du bacille du scepticisme venant de l'ouest », qui avait par ailleurs été si vivement applaudie par l'assemblée qu'il est évident maintenant que Feuchtwanger s'est rendu impopulaire, il va laisser peu d'amis ici. Višnevskij le mettait quasiment au même niveau qu'André Gide. ${ }^{29}$

Mais le public en recevait une toute autre image : dès le début de son voyage, Feuchtwanger avait exigé de faire quelque chose contre Gide. Un article consacré

écrivain danois Martin Andersen-Nexø pendant ses séjours en Union soviétique en 1934 et 1935. Dans l'après-guerre, elle apparaît comme la traductrice entre autres de E.T.A. Hoffmann, Wilhelm Raabe et Herrmann Hesse.

26. Pour plus de détails : Stern, Western Intellectuals, p. 162-174; Idem, « Moscow 1937: The Interpreter's Story », Australian Slavonic and East European Studies 21 (1-2), 2007, p. 73-95. Édition allemande des rapports (traduction et introduction) : Anne Hartmann, «Lion Feuchtwangers Dolmetscherin. Die Rapporte der Dora Karawkina », Exil. Forschung, Erkenntnisse, Ergebnisse 30 (1), 2010, p. 28-51.

27. Rapport D. Karavkina, 11.12.1936. GARF (Gosudarstvennyj Arhiv Rossijskoj Federacii Archives d'État de la Fédération de Russie), Moscou, f. 5283, op. 5, d. 745.

28. Rapport D. Karavkina, 16.12.1936. GARF f. 5283, op. 1, d. 334. « Ainsi, les mots de Gide se trouveraient confirmés, il n'y aurait pas de liberté d'opinion chez vous », se rebella peu après Feuchtwanger parce que la mise à l'impression de son article contre Gide dans la Pravda avait pris du retard et parce que la rédaction demandait des changements. Rapport D. Karavkina, 27.12.1936. GARF f. 5283, op. 8, d. 290.

29. Akademie der Künste (AdK), Berlin, Hugo-Huppert-Archiv Nr. 64-65. Dès le 13 décembre 1936, Aleksandr Arosev, le supérieur de Karavkina, avertit Stalin, Molotov, Ežov et Andreev qu'il s'agirait peut-être dans le cas de Feuchtwanger d'un second Gide. GARF f. 5283, op. 1a, d. 308. «Je me fais des cheveux blancs en pensant à cette idée », commente une rédactrice de la revue Das Wort à Moscou lorsque Feuchtwanger annonce son projet de publier un « petit ouvrage sur la Russie ». Lettre de Julija Šejnina[?] à Maria Osten, 17.3.1937. RGALI f. 631, op. 12, d. 88. 
à sa visite à la rédaction de la Pravda, lui fait décrire Gide comme un esthète amoureux de soi-même qui n'avait jamais été vraiment antifasciste ${ }^{30}$. Peu de temps après, de sa propre initiative, Feuchtwanger relança la polémique de manière plus importante avec l'article «Un esthète en Union soviétique » qui parut le 30 décembre 1936 dans la Pravda, et également dans le numéro de février 1937 de Das Wort $^{31}$. Le Français est caractérisé comme « un Parisien bohème, ergotant et extrêmement égocentrique », ne prenant aucun intérêt à toutes les grandes choses qu'il y avait à voir. Cet esthète blasé avait bien le droit de ne pas trouver l'Union soviétique à son goût, mais la publication de son jugement revenait à « apporter son soutien aux opposants» et par là-même, à porter un coup au «progrès dans le monde ». Feuchtwanger affirma, tout en assumant le style de la campagne soviétique qui commençait alors se développer contre Gide, que l'écrivain français aurait ainsi « perdu le droit de se présenter encore comme un écrivain socialiste». Pour Feuchtwanger cependant, cela ne mettait en aucun cas fin à sa querelle avec Gide. Dans son livre Moskau 1937. Ein Reisebericht für meine Freunde [Moscou 1937. Un récit de voyage pour mes amis], rédigé en mars 1937, Feuchtwanger devait certes retirer l'attaque personnelle, mais il allait conserver la polémique concernant Gide.

Il faut y regarder à deux fois pour s'apercevoir que la vision des choses de Gide et de Feuchtwanger varient au fond très peu l'une de l'autre, mais il est vrai qu'ils classent et exploitent leurs observations de manière différente. Tous deux font par exemple état du bas niveau de vie et de la crise du logement, du manque de produits d'usage courant, du mauvais goût de nombreux articles, des vêtements défraîchis et des longues files d'attente devant les magasins. Ils constatent tous deux, pleins de compréhension, que la demande dépasse de beaucoup l'offre et qu'on ne peut espérer de la qualité qu'après la quantité. Gide plaint cependant ce peuple qui n'a pas d'autre choix et aucun moyen de comparaison, mais auquel on fait croire « qu'on est moins heureux qu'eux partout ailleurs $»^{32}$. Au contraire, pour Feuchtwanger, la « vie heureuse » du peuple soviétique est un fait reposant sur la « méthode lumineuse de l'économie et toute la structure de l'état» qui « consolerait l'individu des manques de la vie privée » et donnerait confiance en l'avenir :

Aussi exactement que les Moscovites savent que le train pour Leningrad part à telle heure, ils savent que dans deux ans ils auront des vêtements, ceux qu'ils voudront et autant qu'ils en voudront, et dans dix ans des appartements, ceux qu'ils voudront et autant qu'ils en voudront. ${ }^{33}$

30. «Lion Fejhtvanger v redakcii "Pravdy" [Lion Feuchtwanger à la rédaction de la Pravda] », Pravda, 15.12.1936.

31. Édition française: «Opinion d'un esthète sur L'Union soviétique », Journal de Moscou, 5.11.1937. Cf. Gaston Bouatchidzé, Retour en URSS avec André Gide. Touches et retouches. P. : Hermann, 2005, p. 206-210.

32. André Gide, Retour de 1'U.R.S.S., P. : Gallimard, 1936, p. 50.

33. Feuchtwanger, Moskau 1937, p. 20 et 21. 
Gide met en garde contre le conformisme, cet « esprit de soumission $»^{34}$ qui serait observable en tous lieux - dans la vie sociale, dans l'activité artistique et dans la pensée politique. Feuchtwanger se voit, à contrecœur, forcé de se ranger à l'avis de Gide, mais il réduit le conformisme à trois points et lui retire ainsi toute sa portée :

l'accord sur les principes fondamentaux du communisme, l'amour commun de l'URSS et l'assurance partagée par tous que l'Union soviétique sera bientôt le pays le plus heureux et le plus puissant du monde. ${ }^{35}$

Une fois encore, il classe les choses sous un angle différent. Tandis que Gide y voit une « tentative de simplification, d'unification, de réduction $»^{36}$, Feuchtwanger voit disparaître « le conformisme si mal famé grâce à l'accroissement de l'éducation» ${ }^{37}$. Sur d'autres points encore, comme la formation d'une nouvelle société de classes et l'apparition d'une mentalité bourgeoise par suite des différences de salaire de plus en plus importantes, sans oublier les traits dictatoriaux de la société soviétique, les observations des deux auteurs ne diffèrent pas dans le fond, mais dans la perspective. Là où, à partir des déficiences observées dans la société soviétique, Gide voit l'expérience soviétique menacée dans ses fondements, Feuchtwanger n'aperçoit que des manques temporaires et faciles à expliquer, qui seront bientôt comblés. Tandis que Gide note depuis la révolution d'Octobre une décadence graduelle, Feuchtwanger entrevoit déjà la silhouette d'un lendemain meilleur à travers l'imperfection du jour présent.

La proximité déguisée avec Gide est une preuve que le livre de Feuchtwanger est plus ambivalent et contradictoire que ne le suggèrent les phrases affirmatives souvent citées. Feuchtwanger a tendance dans son œuvre à laisser une grande place aux sources extérieures (statistiques, informations de seconde main, opinions de ses interlocuteurs, citations littéraires, etc.), qui ont souvent plus voix au chapitre que le « je » du témoin oculaire. Il donne la parole aux « opposants » qui déplorent le « despotisme brutal de Stalin, son goût pour la terreur », son « esprit autoritaire et son désir de vengeance sans limites ${ }^{38}$. Il cite des « sceptiques » et des «douteurs » qui, en rapport avec le deuxième procès-spectacle, insinuent que le régime emploie la torture et le chantage et que la théorie du complot ne serait pas plausible. Tous ces moments sont des indices que l'approbation du régime stalinien dans Moskau 1937 ne repose aucunement sur une certitude absolue. Lorsqu'il déclencha sa polémique contre Gide, Feuchtwanger essaya évidemment en même temps d'atténuer ses

34. Gide, Retour, p. 76.

35. Feuchtwanger, Moskau 1937, p. 48.

36. Gide, Retour, p. 77.

37. Feuchtwanger, Moskau 1937, p. 152.

38. Ibid., p. 103. 
propres doutes ${ }^{39}$. Néanmoins, les différences de ton, d'expression et de déduction entre les deux récits de voyage sont flagrantes. Comment peut-on les expliquer ? Quelques raisons ont déjà été mentionnées, comme le regard plus critique ${ }^{40}$ que Gide portait grâce à la distance avec Moscou et aussi grâce à ses accompagnateurs polyglottes, mais il faut en citer d'autres : l'auteur juif réfugié et isolé de son pays était plus réceptif aux compliments, aux offres lucratives, aux accueils honorifiques et au succès auprès du public que le Français, qui était mentalement et matériellement beaucoup plus indépendant et montrait même de la répugnance pour les privilèges. Feuchtwanger savourait son succès (« À Moscou, on me recevait si triomphalement qu'il était difficile de ne pas se sentir pousser des ailes» ${ }^{41}$ ) alors que Gide, déçu, constatait : «En enthousiaste, en convaincu, j'étais venu pour admirer un nouveau monde, et l'on m'offrait afin de me séduire, toutes les prérogatives que j'abominais dans l'ancien $»^{42}$.

Leurs connaissances antérieures et leurs attentes étaient également tout à fait différentes : tandis que Gide avait été avant tout attiré par « la forêt [...] affreusement touffue [...] des questions sociales $»^{43}$ et la promesse humaine d'une nouvelle société, le voyage de Feuchtwanger se déroulait sous le signe de la mobilisation contre Hitler. Il est vrai que de 1933 à 1935, André Gide avait soutenu de nombreuses actions et présidé des associations et des congrès antifascistes, mais ses doutes s'étaient déjà amplifiés avant le voyage, par exemple à cause de l'affaire Victor Serge, mais aussi à cause de la législation soviétique contre l'homosexualité. «L'éclatante lumière qui tirait nos regards vers la Russie » avait ainsi considérablement pâli avant même qu'il n'entame son voyage ${ }^{44}$. En 1936, Feuchtwanger venait au contraire de renforcer son engagement envers l'Union soviétique, et pas

39. Cf. Wulf Köpke, « Das dreifache Ja zur Sowjetunion. Lion Feuchtwangers Antwort an die Enttäuschten und Zweifelnden », in Exilforschung: Ein internationales Jahrbuch, vol. 1, München : Text + Kritik, 1983, p. 61-72 ; Anne Hartmann, « Abgründige Vernunft - Lion Feuchtwangers Moskau 1937 », in Norbert Otto Eke, Gerhard P. Knapp, éds., Neulektüren New Readings. Festschrift für Gerd Labroisse zum 80. Geburtstag, Amsterdam : Rodopi, 2009, p. 149-177.

40. Les notes de voyage de Gide font aussi preuve de sa curiosité et de sa sensibilité : « [Carnets d'U.R.S.S.] » in Martine Sagaert, éd., Journal II : 1926-1950, P. : Gallimard, 1997, p. 523-540. Cf. Martine Sagaert, « André Gide et la Russie soviétique ou de l'usage de la retouche », in Idem, Peter Schnyder, André Gide. L'écriture vive, Bordeaux : Presses Universitaires, 2008, p. 35-53. Les notes - inédites - de Feuchtwanger contiennent par contre peu de réflexions sur les choses vécues.

41. Lettre de Lion Feuchtwanger à Arnold Zweig, 9.12.1936, in Harold von Hofe, éd., Lion Feuchtwanger - Arnold Zweig: Briefwechsel 1933-1958, Frankfurt/M. : Fischer, 1986. Vol. I, p. 122.

42. Gide, Retouches, p. 59-60.

43. Gide, Retour, p. 33.

44. Lettre d'André Gide à Claude Naville, 10.9.1935, citée par Bernard Duchatelet, Romain Rolland et la N.R.F. (Cahiers Romain Rolland n² 27), P. : Albin Michel, 1989, p. 65. Cf. Mazuy, Croire plutôt que voir ?, p. 157. 
seulement en tant qu'éditeur d'une revue publiée à Moscou ${ }^{45}$. Il était parti lui aussi avec quelques craintes, mais elles concernaient moins la situation du pays que la renommée de celui-ci à l'ouest. Ainsi, il demanda à rencontrer d'urgence Dimitrov pour « lui parler du procès des trotskistes, car ce procès aurait bouleversé toute l'Europe et aurait coûté deux tiers de ses partisans à l'Union soviétique $»^{46}$. À Moscou, Feuchtwanger était resté sceptique face aux arguments de Dimitrov, de Tal' et des autres, mais dans son livre sur Moscou, il se prétendait convaincu.

Qu'est-ce qui avait déclenché ce revirement ? La conversation avec Stalin futelle décisive, comme le suppose Ludmila Stern $?{ }^{47}$ Cette hypothèse semble convaincante, mais jusqu'à ce jour il est impossible de la prouver. Le dernier rapport de Dora Karavkina date du 4 janvier, de sorte qu'il n'y a pas de notes de l'interprète pour les quatre semaines qui suivirent cette date, y compris pour le jour de l'entretien (8 janvier). Ni la correspondance de Feuchtwanger à Moscou ni son journal intime qu'il tenait quotidiennement n'apportent d'éclaircissements sur son attitude politique ou bien son changement d'opinion. Il y a certainement eu plusieurs raisons qui l'ont poussé à écrire son livre « probolchevik ${ }^{48}$. Sans doute, l'entretien avec Stalin $^{49}$ fit-il grande impression sur Feuchtwanger. L'émigré qui, à l'époque, était si souvent considéré comme un paria social et solliciteur embarrassant, se trouvait élevé au rang d'acteur politique de premier ordre. Ce qui avait surtout fasciné Feuchtwanger quant au contenu, c'était le scénario d'un vaste complot que Stalin lui avait exposé dans un soudain mouvement de colère, après avoir donné des explications interminables et redondantes sur le rôle de l'écrivain dans la société soviétique, la situation sociale de l'intelligentsia, etc.

La portée de cette conversation fut énormément renforcée par le fait que Stalin, en tant que plus haute instance politique, confirmait et certifiait pour ainsi dire la version de la situation en Union Soviétique qui avait déjà été donnée à Feuchtwanger par ses autres interlocuteurs soviétiques. L'écrivain allemand s'était laissé envoûter par une vision du monde homogène ainsi que par une rhétorique uniforme, ce qui devait avoir un fort pouvoir de séduction. Cet effet fut encore renforcé lorsque Feuchtwanger, cet homme de théâtre, put observer de ses propres yeux le

45. Mais il ne connaissait pas en détail le développement politique en URSS. « Je vois à quel point je suis peu informé », confiait-t-il dans son journal intime le 8 mars 1937 en rédigeant son récit de voyage.

46. Rapport D. Karavkina, 17.12.1936. GARF, f. 5283, op. 1, d. 354.

47. Stern, Western Intellectuals, p. 171; Idem, « Moscow 1937 », p. 88. Dans son « casestudy » sur Feuchtwanger Stern se limite exclusivement à des documents russes, notamment les rapports de Karavkina. Elle ne prend cependant pas en considération des sources germanophones ou le contexte de l'émigration.

48. Lettre de Feuchtwanger à Willi Münzenberg, 8.9.1937. FML. Special Collections, Box C2-c (Correspondence with other writers, Mar-O).

49. RGASPI, f. 558, op. 11, d. 820. Première publication russe : Maksimenkov, « Očerki nomenklaturnoj istorii sovetskoj literatury... », čast' I, p. 250-270. Traduction allemande de l'entretien et reconstruction du séjour de Feuchtwanger à Moscou : Anne Hartmann, « Lost in translation. Lion Feuchtwanger bei Stalin, Moskau 1937 », Exil. Forschung, Erkenntnisse, Ergebnisse 28 (2), 2008, p. 5-31. 
procès-spectacle de Radek, Pjatakov, et bien d'autres ${ }^{50}$. Au début, d'après ce qu'il a consigné dans son journal intime, Feuchtwanger trouvait cependant le procès « très éprouvant » (23 janvier), « assez insipide» (26 janvier), «pas très intéressant » ( 27 janvier). Son sentiment ne changea qu'au moment des aveux : «Les discours des accusés sont impressionnants », commente-t-il le 29 janvier. Tout comme d'autres observateurs de l'ouest ayant assisté au procès, Feuchtwanger finit par succomber à la « redoutable force des apparences $»^{51}:$ «sous l'influence de ce que ces accusés disaient et de la façon dont ils le disaient, mes doutes [s'évaporèrent], comme le sel se dissout dans l'eau $»^{52}$.

Par ailleurs, Feuchtwanger prenait position dans la presse soviétique. En plus des attaques contre Gide, il publia deux articles dans lesquels il approuvait en principe le tribunal et empruntait à nouveau la diction soviétique. «Il est hors de doute que la culpabilité des accusés est prouvée sans équivoque», affirme-t-il. «Tous ceux qui ne sont pas de mauvaise foi, doivent avouer que l'origine idéologique, et souvent matérielle, de leurs actes revient à Trotskij ${ }^{53}$. Ces paroles, qui avaient immédiatement été rapportées dans la presse communiste allemande, suscitèrent peu après de vives critiques à l'ouest. Le 12 février 1937, des écrivains norvégiens connus adressèrent une lettre ouverte à Feuchtwanger, lui reprochant de défendre une action judiciaire qui équivalait à une «exécution des innocents » ${ }^{54}$. Le 15 février, Klaus Mann écrivit dans son journal intime : «Des journaux communistes avec un commentaire enragé et fatal de Feuchtwanger sur le procès de Moscou $»^{55}$. Feuchtwanger se plaignit du

50. Feuchtwanger fut probablement invité par Stalin personellement, en tout cas sa participation au procès fut autorisé par le bureau politique. Cf. Postanovlenie politbjuro CK VKP(b) «O processe po delu Pjatakova, Radeka, Sokol'nikova, Serebrjakova i dr. [Décret du Bureau politique du CC du PCP(b). «Du procès Pjatakov, Radek, Sokol'nikov, Serebrjakov et autres »] », in V.N. Haustov [et al.], éds., Lubjanka. Stalin i glavnoe upravlenie gosbezopasnosti 1937-1938 [La Lubjanka. Stalin et l'administration principale de la Sécurité d'État], M. : MFD 2004, p. 55.

51. Ernst Fischer, Erinnerungen und Reflektionen, Frankfurt/M. : Büchergilde Gutenberg, 1994, p. 422.

52. Feuchtwanger, Moskau 1937, p. 119.

53. Lion Fejhtvanger, « Pervye vpečatlenija ob ètom processe [Premières impressions de ce procès]», Pravda, 30.1.1937. Cf. «Lion Fejhtvanger o pervom dne processa, [Lion Feuchtwanger sur le premier jour du procès] », Izvestija, 25.1.1937. Toutefois il gardait quelques doutes : «Les raisons ultimes des actions des accusés et surtout les raisons ultimes de leur comportement au tribunal ne sont cependant pas devenues suffisamment plausibles pour les gens de l'ouest », déclare-t-il dans la Pravda. Le fait que la presse soviétique publiait cette prise de position, qu'il cite dans son livre Moskau 1937 (p. 134), était pour Feuchtwanger l'argument décisif contre la critique de Gide. Cf. Lettre de Feuchtwanger à Zweig, 15.4.1937, in von Hofe, éd., Lion Feuchtwanger - Arnold Zweig. Briefwechsel I, p. 156.

54. La lettre, parue d'abord dans le journal Arbeiterbladet, 12.2.1937, est signée par les écrivains Nini Roll Anker, Lars Berg, Sigurd Hoel, Gunnar Reiss-Andersen, Aksel Sandemose, Nils Collet Vogt, Arnulf Oeverland. Feuchtwanger Articles. La lettre fut ensuite publiée « dans la plupart des journaux scandinaves et beaucoup des journaux anglais », mais aussi « dans quelques parties de la presse des émigrés ». Lettre de Feuchtwanger à Willi Bredel, 8.3.1937, in von Hofe, Washburn, éds., Lion Feuchtwanger. Briefwechsel mit Freunden I, p. 388.

55. Joachim Heimannsberg, éd., Klaus Mann, Tagebücher 1936 bis 1937, Munich : Spangenberg, 1990, p. 107. Dès le 14 février, lors de la lecture du Dossier du procès, Mann s'était demandé : « De quelle façon a-t-on réalisé ces auto-accusations fantastiques ? (ibid.). 
《braillement hystérique $»^{56}$ avec lequel il avait été accueilli à Paris à cause de ses prises de position à Moscou. À son retour, Feuchtwanger se sentait «énormément agoni d'injures [...], et ce de toutes parts $»^{57}$. Comme il résume plus tard dans une lettre à Brecht, les cent lignes qu'il avait publiées à Moscou - sous-entendu « les quatre-vingts sur Gide et les vingt sur le procès »- lui avaient valu « bien plus d'invectives que Erfolg ou Les Oppermann $»^{58}$. Il s'agissait donc bien d'une réaction d'obstination lorsqu'il décida « d'écrire un petit livre, un peu plus volumineux que celui de Gide, sur mes impressions à Moscou $»^{59}$.

La portée biographique des deux voyages était également différente. Tandis que Gide pouvait retrouver sa maison et son ancienne vie, pour Feuchtwanger le propre avenir et le destin de l'Allemagne étaient liés d'une façon décisive à l'estimation de l'Union soviétique. Il est donc logique que les deux écrivains aient abouti à des conclusions divergentes dans la rationalisation politique des expériences qu'ils avaient faites en URSS et des conséquences à en tirer pour l'opinion publique. Gide décida de rompre «explicitement le pacte d'autocensure ${ }^{60}$; il rendit sa critique publique, même si elle n'était pas opportune et même s'il fournissait ainsi des munitions à ses adversaires politiques. Au contraire, Feuchtwanger limita ou relativisa ses doutes afin d'aider l'Union soviétique dans son « combat contre ses nombreux ennemis ${ }^{61}$. Il était même prêt à adapter ses écrits aux souhaits des Soviétiques. En mai 1937, Kol'cov lui rendit visite à Sanary, alarmé par son projet d'un livre sur l'URSS $^{62}$. « Kol'cov est là », note Feuchtwanger dans son journal intime le 23 mai : «Il plaît beaucoup à Marta. Bavarde vivement avec lui. Il trouve les passages sur Trotskij dans mon ouvrage sur la Russie dangereux. »

Durant les jours qui suivirent, Feuchtwanger retoucha entièrement le sixième chapitre «Stalin et Trotskij », « avec assez de mauvaise conscience » (26 mai), mais

56. Lettre de Feuchtwanger à Zweig, 24.2.1937, in von Hofe, éd., Lion Feuchtwanger - Arnold Zweig. Briefwechsel I, p. 149.

57. Lettre de Feuchtwanger à Willi Bredel, 8.3.1937. Cf. Lettre de Feuchtwanger à Eva Herrmann, 24.2.1937. Deutsches Literaturarchiv Marbach.

58. Lettre de Feuchtwanger à Bertolt Brecht, 27.3.[1937], in von Hofe, Washburn, éds., Lion Feuchtwanger: Briefwechsel mit Freunden I, p. 35.

59. Lettre de Feuchtwanger à Willi Bredel, 8.3.1937. Feuchtwanger publia l'introduction comme pré-publication déjà le $1^{\text {er }}$ avril: « Ein Reisebericht », Die neue Weltbühne 33 (14), 1937 p. $418-421$.

60. Sophie Coeuré, La Grande lueur à l'est : Les Français et l'Union soviétique 1917-1939, P. : Éditions du Seuil, 1999, p. 227.

61. Feuchtwanger, Moskau 1937, p. 13.

62. L'insuccès avec l'auteur français était surtout attribué à Kol'cov qui avait invité Gide (et Feuchtwanger) et qui l'avait accompagné pendant son voyage à travers l'Union. En 1938, on lui reprocha d'avoir influencé Gide dans un sens antisoviétique. « Specsoobščenie N.I. Ežova i L.P. Berija I.V. Stalinu s priloženiem sledstvennyh materialov na M.E. Kol'cova, 27.9.1938 [Message confidentiel de N.I. Ežov et L.P. Berija à Stalin avec des éléments d'enquête sur M.E. Kol'cov] », in Haustov [et al.], éds., Lubjanka, p. 558. Cf. Viktor Fradkin, Delo Kol'cova [L'affaire Kol'cov], M. : Vagrius, 2002, p. 90-93, 210-218. Peu après la disparition de Kol'cov - il fut arrêté en décembre 1938, fusillé en février 1940 - la revue Das Wort qui était sous sa protection dut mettre un terme à sa publication. 
malgré tout avec une obligeance remarquable, bien qu'il ait dû modifier les épreuves de son livre : l'hommage aux mérites et aux qualités de Trotskij fut supprimé à la réécriture, pour mettre en relief la «supériorité » de Stalin ; les passages critiques, surtout concernant la politique publique de la culture et des arts, furent édulcorés ; le texte fut amputé de toutes les phrases qui établissaient un lien insidieux entre l'Union soviétique de Stalin et l'Allemagne nazie ${ }^{63}$. Les modifications sont consternantes et ont une grande portée ; ainsi Feuchtwanger raya-t-il, sans le remplacer, un passage dont l'annotation dans la marge « On n'efface pas l'histoire avec une gomme» constituait pour un écrivain historique une règle éthique que l'auteur n'hésita pas à enfreindre lui-même :

Cet homme, Léon Trotskij, est aujourd'hui mis au ban de la société soviétique, et là-bas, on préférerait pouvoir rayer de l'histoire les pages qu'il a écrites. Mais cela est impossible ; l'affaire Trotskij ne sera bouclée et les esprits soviétiques pacifiés que lorsqu'on fera preuve d'assez de justice pour replacer Trotskij dans le contexte historique de son époque. ${ }^{64}$

Cette autocensure n'était peut-être que la conséquence du double objectif que Feuchtwanger s'était fixé avec son livre. D'une part, il voulait absolument que les Soviétiques aussi lisent Moskau 1937-et cela même au prix de larges concessions. D'autre part, il voulait combler la brèche dans le front antifasciste que le récit de voyage de Gide avait, selon lui, causé.

Tandis que Gide se considérait comme l'avocat de la « vérité », Feuchtwanger, lui, se positionnait comme le héraut d'une mission politique en mettant son récit de voyage au service d'une cause plus importante. « Leur cœur nie, ce que leur cerveau affirme », dit Feuchtwanger en condamnant ces intellectuels qui étaient, en théorie, des socialistes, mais qui, par leur comportement, aidaient l'ordre capitaliste. En tant «qu'écrivain porteur d'une certaine responsabilité » ${ }^{65}$ il défendit contre les anciens sympathisants à l'ouest qui « tombaient des nues à cause de ces procès arbitraires et violents », l'idée que l'Union soviétique était « la dernière chance de la civilisation contre la décadence $»^{66}$. Ce qui l'intéressait, c'était « la performance historique de portée mondiale qui était accomplie là-bas », pas la vie quotidienne concrète ou l'individu en tant que tel. Dans une telle perspective, la fin justifie les moyens, et par-là même, l'emploi de la violence : de toute manière on ne pourrait pas « faire l'histoire en mettant des gants ${ }^{67}$. Il défendait ainsi, pour citer Max Weber, une « éthique de la responsabilité », opposée à « l'éthique de conviction »

63. Cf. Anne Hartmann, « Lion Feuchtwanger, zurück aus Sowjetrussland. Selbstzensur eines Reiseberichts », Exil. Forschung, Erkenntnisse, Ergebnisse 29 (1), 2009, p. 16-33.

64. FML, Special Collections, Box D 12 : Lion Feuchtwanger, Moskau 1937 [manuscrit, versions, épreuves].

65. Feuchtwanger, Moskau 1937, p. 149.

66. Ibid., p. 144.

67. Ibid., p. 147. 
incarnée par Gide ${ }^{68}$. Le « cas » Gide-Feuchtwanger illustre parfaitement le dilemme des intellectuels du $\mathrm{Xx}^{\mathrm{e}}$ siècle : conflit personnel entre esprit et pouvoir, conscience morale et prise de parti, repli sur soi et engagement ${ }^{69}$.

Alors que dans ses romans, Feuchtwanger cherchait toujours à « rendre différentes perspectives explicites et plausibles » sans donner gain de cause à un seul personnage, dans son récit de voyage, il ne laissait que peu de place aux ambivalences $^{70}$. Le concept de vérité doit reculer devant celui de raison qui lui sert comme maxime dans son écriture et qu'il considère en même temps comme l'un des fondements de l'État soviétique. Le « combat mené par une minorité dotée de raison contre une majorité d'imbéciles ${ }^{71}$, ce qui pour Feuchtwanger était une constante dans l'histoire du monde, aboutissait selon lui à une victoire de la raison en URSS. La façon dont Feuchtwanger conçoit ici la raison nous ramène moins au concept d'émancipation de l'époque des Lumières, qu'à celui des utopies d'un État idéal, comme celles de Morus ou de Campanella. Son aspiration à un « consensus omnium $\gg^{72}$ est en ce sens véritablement utopique (et opposée au pluralisme et à l'individualisme modernes), même si placer le bien de tous au-dessus des intérêts individuels signifie l'élimination de ceux qui perturbent l'équilibre harmonieux. Mais l'adjuration de Feuchtwanger en faveur d'une « raison géométrique » et «d'une éthique sobre » reste froide et stérile. Contre la volonté de l'auteur, le revers totalitaire du « meilleur des mondes » se fait jour, ainsi que le désaccord entre le cœur et le cerveau, pour conserver sa terminologie.

Les concessions faites à la vérité et aux émotions ne payèrent pas. Il est vrai que Feuchtwanger était satisfait des premiers effets des éditions anglaise et américaine et qu'il espérait «avoir ainsi compensé une bonne partie des dommages causés par Gide » :

Tout du moins, c'est ce qu'on m'écrit avec conviction depuis les quatre coins du monde. [...] Même des journaux connus pour être hostiles à l'Union soviétique tels que le Times ou le Manchester Guardian constatent que l'on ne peut résister au pouvoir de persuasion de nombre de mes arguments. ${ }^{73}$

68. Max Weber, Politik als Beruf(1919), Berlin : Dunker \& Humblot, 1958, p. 57-67.

69. Voir notamment Michel Winock, Le siècle des intellectuels, P. : Éditions du Seuil, 1999. Sur les Allemands exilés en France : Albrecht Betz, Exil et engagement : Les intellectuels allemands et la France 1930-1940, P. : Gallimard, 1991.

70. Martina Winkler, « Das Dilemma intellektuellen Engagements oder Der Fluch erfüllter Wünsche: Lion Feuchtwangers "Moskau 1937" », in Idem, éd., WortEnde. Intellektuelle im 21. Jahrhundert?, Leipzig : Universitäts-Verlag, 2001, p. 90 et 93.

71. Feuchtwanger, Moskau 1937, p. 8.

72. Ibid., p. 51. Pour sa recherche du consensus, cf.: ibid., p. 96-103 ; Martin Sabrow, « Consent in the Communist GDR or How to interpret Lion Feuchtwanger's Blindness in Moscow 1937 », in Paul Corner, éd., Popular Opinion in Totalitarian Regimes: Fascism, Nazism, Communism, Oxford : UP, 2009, p. 168-183.

73. Lettre de Feuchtwanger à Maria Osten, 24.8.1937. - RGALI, f. 631, op. 13, d. 87. 
Feuchtwanger fut comblé lorsqu'en novembre 1937 parut une traduction en russe à grand tirage (《le livre a fait grande sensation là-bas $»^{74}$ ), bien que cette édition dût bientôt disparaître des librairies et des bibliothèques soviétiques ${ }^{75}$. En France néanmoins, il lui fut impossible de publier sa réponse à Gide, alors qu'elle y aurait été «bien plus nécessaire que dans les pays anglophones », «mais cela était hors de question pour mon éditeur bourgeois $\gg^{76}$. La version allemande, que les éditions Querido d'Amsterdam ne publièrent pas sans scrupules, ne contribua pas non plus à la « compréhension des choses russes » (29.3.1937) comme Feuchtwanger le souhaitait, mais intensifia au contraire les tensions parmi les émigrés allemands. Seuls furent convaincus ceux qui l'étaient déjà (Bertolt Brecht, Ernst Bloch, Heinrich Mann), les sceptiques restèrent dans le doute (Arnold Zweig, Klaus Mann) tandis que les critiques de l'Union soviétique (Leopold Schwarzschild, Kurt Hiller) prirent de plus en plus de distance avec Feuchtwanger et le front populaire.

Feuchtwanger dut réaliser que son récit de voyage n'avait pas eu l'effet escompté, ni à l'ouest, ni en URSS. S'il observa avec satisfaction les succès initiaux des deux côtés, il cessa quasiment par la suite de faire allusion à son livre sur la Russie. Jusqu'au printemps 1938, il envisageait encore de retourner en Union soviétique et de visiter d'autres villes et régions, plus tard il n'en fut plus question. Déjà depuis l'été 1937, l'engagement de Feuchtwanger en faveur de l'Union soviétique et du front populaire allemand avait tiédi, la situation parmi les émigrés gauchistes étant surtout de plus en plus compliquée et perturbée.

Chacun tire la couverture à soi, personne ne veut écouter l'autre, et quand on veut proposer un programme applicable, on n'obtient que des élucubrations confuses et idéologiques. ${ }^{77}$

Feuchtwanger, qui avait fait son entrée dans la vie politique pour ainsi dire contre son gré, se retirait des affaires politiques et ne perdait plus son temps à débattre dans des congrès. Dans son roman Exil (1940), qui repose principalement sur son expérience en URSS, il renonce de nouveau à toute univocité idéologique et présente des points de vue divergents. Le jeune Hanns Trautwein quitte la

74. Lettre de Feuchtwanger à Eva Hoboken, 2.12.[1937], in Nortrud Gomringer, éd., Lion Feuchtwanger: Briefe an Eva van Hoboken, Wien : Ed. Splitter, 1996, p. 176. Dans la version russe le traducteur n'est pas mentionné ; mais de par une lettre de Karavkina à Feuchtwanger (3.12.1937) il est clair que c'est elle qui a traduit le livre. - RGALI, f. 631, op. 12, d. 79.

75. On a souvent soupçonné, que la traduction russe avait été victime de la censure. Il semble cependant que le retrait ne soit pas le résultat d'une décision politique, mais celui d'un excès de zèle de quelques fonctionnaires. Dans une circulaire, A. Samohvalov, sousdirecteur de l'agence de la censure, indiqua le fait, que « les livres de Feuchtwanger ne sont nullement soumis à la confiscation. » «A. Samohvalov vsem načal'nikam glavlitov, krajoblitov [A. Samohvalov aux chefs des administrations principaux et régionaux de la littérature et des maisons d'édition]», 27.1.1938. - GARF, f. 9425, op. 1, d. 312.

76. Lettre de Feuchtwanger à Münzenberg, 8.9.1937.

77. Lettre de Feuchtwanger à Heinrich Mann, 28.10.1937, in von Hofe, Washburn, éds., Lion Feuchtwanger. Briefwechsel mit Freunden I, p. 329. 
communauté parisienne des émigrés d'Allemagne pour l'Union soviétique afin d'y faire des études d'architecture et de bâtir un monde nouveau. Il a pour credo que 《la seule réponse à la violence est la violence ${ }^{78}$. Son père Sepp, un « libéral incorrigible $»^{79}$, doit certes s'avouer vaincu face aux arguments purement rationnels de son fils, mais ses objections pèsent aussi dans la balance : «La fin ne justifie pas les moyens, mais les moyens peuvent entacher la fin »; une politique socialiste qui ne s'occupe pas de l'individu constitue une "caricature du socialisme ${ }^{80}$ et : ce n'est pas parce que l'on dit non à Hitler que l'on doit dire oui à l'Union soviétique. Finalement, tous les deux arrivent à la conclusion que pour Sepp, inapte à la grande politique, l'art « est la seule manière [...] de participer au grand combat $»^{81}$. C'est apparemment sur cette prise de conscience que Feuchtwanger va s'appuyer pour la suite de son œuvre.

Seminar für Slavistik / Lotman-Institut für russische kultur, Ruhr-Universität Bochum

Anne.Hartmann@ruhr-uni-bochum.de

78. Feuchtwanger, Exil, p. 195.

79. Ibid., p. 559.

80. Ibid., p. 836.

81. Ibid., p. 977. 